Article

\title{
Synthesis of Peptide-Immobilized Magnetic Beads, and Peptide Reactivity Assay for Assessing Skin Sensitization Utilizing Chromophore
}

\author{
Hiroshi Miyazaki ${ }^{1}$, Hikaru Takaishi $^{2}$, Hidefumi Ikeda $^{3}$, Hideto Ariumi $^{4} \oplus$, Yoshio Hamada ${ }^{2}$, \\ Kunihiko Yamashita ${ }^{1, *}$ and Kenji Usui ${ }^{2, *(D)}$ \\ 1 Medical Device Division, Innovation and Business Development Headquarters, Daicel Corporation, \\ Minato-ku, Tokyo 108-8230, Japan; hs_miyazaki@jp.daicel.com \\ 2 Faculty of Frontiers of Innovative Research in Science and Technology (FIRST), Konan University, Chuo-ku, \\ Kobe 650-0047, Japan; s1691023@a.konan-u.ac.jp (H.T.); pynden@gmail.com (Y.H.) \\ 3 Product Assurance Division, Mandom Corporation, Chuo-ku, Osaka 540-8530, Japan; \\ hidefumi.ikeda@mandom.com \\ 4 Faculty of Pharmaceutical Sciences, Sanyo-Onoda City University, Sanyo-Onoda, \\ Yamaguchi 756-0884, Japan; ariumih@rs.socu.ac.jp \\ * Correspondence: ku_yamashita@jp.daicel.com (K.Y.); kusui@konan-u.ac.jp (K.U.)
}

Received: 4 September 2020; Accepted: 1 October 2020; Published: 7 October 2020

\begin{abstract}
DPRA (direct peptide reactivity assay) and ADRA (amino acid derivative reactivity assay), which are based on the biological events of skin sensitization, were developed as alternatives to the controversial animal experiments. These assays are described in the OECD (Organization for Economic Co-operation and Development) guideline, Test No. 442C. Although these assays have been endorsed by the industries and internationally accepted as promising and effective tests for in vitro skin sensitization, they suffer from several drawbacks, such as incompatibility with hydrophobic chemicals and complicated sample processing. Here, we demonstrated a chromophore-based solid phase peptide reaction assay in vitro using peptides immobilized on magnetic beads (C-SPRA-MB). We successfully synthesized lysine (Lys) and cysteine (Cys) immobilized on magnetic microbeads. However, Cys immobilized magnetic microbeads showed gradual decomposition of the magnetic beads due to $\mathrm{SH}$ oxidation. Using Lys immobilized magnetic microbeads, we demonstrated the capacity of C-SPRA-MB to predict skin sensitization by measuring free amino groups of the Lys after reaction with test chemicals. First, the free amines on the microbeads were reacted with bromophenol blue (BB). Then, by treatment with a saturated solution of Lys, the bound BBs were released and quantified. C-SPRA-MB provides high-throughput and accurate assays for assessments of chemicals, including with low-potency as skin sensitizers and poor water solubility. C-SPRA-MB may be useful for effective prediction of their skin sensitization potential in the process of compound screening, especially in the case of misclassified by DPRA and ADRA. Thus, C-SPRA-MB can be applied to assessing the sensitization potential of medical, pharmaceutical, cosmetics, and industrial compounds.
\end{abstract}

Keywords: skin sensitization; alternatives to animal testing; peptide synthesis; magnetic beads; DPRA

\section{Introduction}

The potential to trigger skin sensitization must be effectively assessed for various chemical compounds present in industrial, cosmetics and pharmaceutical products to minimize the risk of developing skin diseases. Animal experiments, including local lymph node assay (LLNA) [1], were widely used to assess the sensitization potential of chemical compounds. However, animal experiments for assessing such risk potential, especially in cosmetics, were prohibited in the EU 
in 2011 [2]. Therefore, in vitro alternatives to animal testing for sensitization are indispensable. The biological processes underlying skin sensitization are well understood, and the covalent binding of a hapten to skin protein is the key event [3]. Based on this, DPRA (direct peptide reactivity assay) $[4,5]$ and ADRA (amino acid derivative reactivity assay) [6-8] were developed as promising in vitro skin sensitization tests. They are even described in the OECD (Organization for Economic Co-operation and Development) guideline, Test No. 442C [9]. DPRA and ADRA use HPLC and are based on the reactivity of a sensitizer with either Lys ( $K$, lysine) or Cys ( $C$, cysteine). Although these two test methods are internationally accepted and endorsed by industries as promising and effective in vitro skin sensitization tests, they have several drawbacks [10-12]: (i) candidate drugs or cosmetic chemicals often have poor water-solubility and thus do not dissolve in polar solvents used in the assays; (ii) an unreacted test compound and an unreacted peptide may be co-eluted in the same retention time resulting in overlapping their peaks; (iii) Cys-peptide can easily dimerize via its free thiol upon oxidation, potentially leading to false positives; (iv) HPLC is relatively difficult handling and time-consuming; (v) because DPRA and ADRA are kinds of an oversimplified chemical assay, specific information, including reaction mechanisms, kinetics and detailed reactivity, etc., cannot be obtained.

To overcome these limitations, we proposed the C-SPRA (chromophore-solid phase peptide reaction assay) for assessing in vitro sensitization [13]. As polystyrene beads are used in C-SPRA as the solid phase and they require manual manipulation, it is difficult to analyze multiple samples simultaneously. Thus, in this study, we report the utilization of magnetic beads as solid support to immobilize peptides to develop a modified C-SPRA for higher-throughput analysis (Figure 1). Magnetic beads are easier to handle and are more versatile. Since the use of magnetic beads allows automation, a larger volume of data can be generated rapidly. Here, we describe implementation of C-SPRA method using magnetic microbeads (C-SPRA-MB) and demonstrate its use for assessment of the skin sensitization potential of chemical compounds in vitro.

(a)
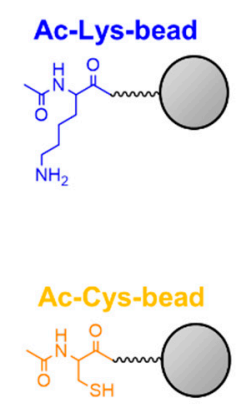

(b)

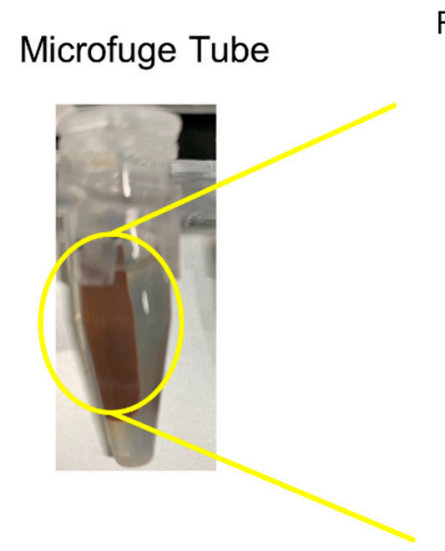

Ac-Lys-beads or Ac-Cys-beads
Removal of unreacted chemicals

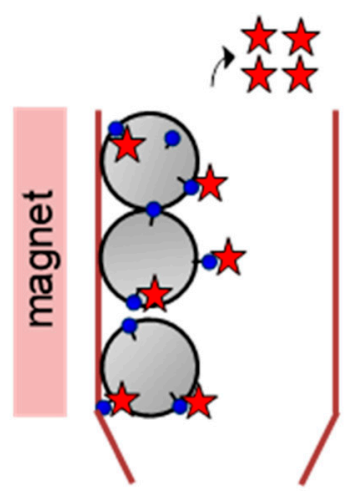

Chemical

Figure 1. (a) Structures of Ac-Lys beads and Ac-Cys beads. (b) An illustration of the column used in chromophore-based solid phase peptide reaction assay in vitro using peptides immobilized on magnetic beads (C-SPRA-MB).

\section{Materials and Methods}

\subsection{Test Chemicals and Materials}

The test chemicals were p-benzoquinone (BQ), fluorescein-5-isothiocyanate (FITC), benzylidene acetone (BA), undec-10-enal (UE), 5-methyl-2-phenyl-2-hexanal (MPH), $\alpha$-amyl cinnamic aldehyde (ACA), and dibutyl phthalate (DP). All test chemicals were purchased from FUJIFILM Wako Pure 
Chemical Corporation (Osaka, Japan). Special grade reagents were used. The test chemicals were dissolved in HPLC grade dimethyl sulfoxide (DMSO) and N-methylmorpholine. All chemicals were used without further purification.

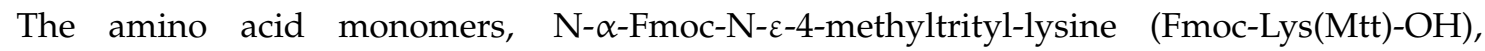
Fmoc-4-methyltrityl-cysteine (Fmoc-Cys(Mtt)-OH), and Fmoc-3-amino-3- (2'-nitrophenyl) propionic acid (Fmoc-npp-OH) were used. The amino acids, 2-(1H-benzotriazole-1-yl)1,1,3,3-tetramethyluronium hexafluorophosphate (HBTU) and 1-hydroxy benzotriazole monohydrate $(\mathrm{HOBt})$ were purchased from Watanabe Chemical Industries, Ltd (Hiroshima, Japan), and 5(6)-carboxyfluorescein was purchased from FUJIFILM Wako Pure Chemical Corporation.

\subsection{Preparation of Fmoc-Lys(Mtt)-npp Beads and Ac-Lys(Flu)-npp Beads}

Fmoc-Lys(Mtt)-npp beads and fluorescein tagged Ac-Lys(Flu)-npp beads were prepared on micromer-M PEG- $\mathrm{NH}_{2}$ (micromod Partikeltechnologie $\mathrm{GmbH}$ ) by manual synthesis using Fmoc chemistry [14] with a magnetic tube holder and separator (DynaMag-2, Thermo Fisher). Coupling of the amino acids were carried out using HBTU and HOBt (200 eq.). First, Fmoc-npp-OH was coupled to the magnetic microbeads, and then Fmoc-Lys(Mtt)-OH was coupled. For checking the purity, Fmoc-Lys(Mtt)-npp beads in a test tube were irradiated with UV light $(365 \mathrm{~nm}, 25 \mathrm{~W})$ for $10 \mathrm{~min}$. Then, the solution was analyzed by HPLC using a GL-7400 HPLC system (GL Sciences, Tokyo, Japan), an Inertsil ODS-3 column ( $4.5 \times 150 \mathrm{~mm}$; GL Science), and a linear acetonitrile/0.1\% trifluoroacetic acid (TFA) gradient at a flow rate of $1.0 \mathrm{~mL} / \mathrm{min}$. The eluate was monitored at $301 \mathrm{~nm}$ for the absorbance of the Fmoc moiety. After removal of the Fmoc group from Fmoc-Lys(Mtt)-npp beads, the N-terminal free amine was acetylated with acetic anhydride in DMSO to obtain Ac-Lys(Mtt)-npp beads. The Mtt group was removed from the sidechain by incubating Ac-Lys(Mtt)-npp beads for $2 \mathrm{~h}$ in Mtt deprotection solution (DMSO/dichloromethane/trifluoroacetic acid (TFA)/triisopropylsilane $(47: 47: 1: 5, v / v))$, and then Ac-Lys-npp beads were washed 10 times with Mtt deprotection solution and DMSO. Following this, the liberated free amine on the sidechain of Ac-Lys-npp beads was coupled to 5(6)-carboxyfluorescein to obtain, Ac-Lys(Flu) beads. To confirm Mtt deprotection, Ac-Lys(Flu) beads were irradiated with UV light for $10 \mathrm{~min}$, and then the solution was analyzed by HPLC. The eluate was monitored at $490 \mathrm{~nm}$ for the absorbance of fluorescein moiety.

\subsection{Preparation of Ac-Lys Beads and Ac-Cys Beads}

Fmoc-Lys(Mtt)-OH and Fmoc-Cys(Mtt)-OH were coupled directly to the magnetic microbeads. After removal of Fmoc from Fmoc-Lys(Mtt) beads and Fmoc-Cys(Mtt) beads, the N-terminal free amine was acetylated using acetic anhydride in DMSO to obtain Ac-Lys(Mtt) beads and Ac-Cys(Mtt) beads, respectively. Mtt groups present in the sidechains of Lys and Cys were removed by incubating the Ac-Lys(Mtt)-npp beads and Ac-Cys(Mtt)-npp beads for $2 \mathrm{~h}$ in Mtt deprotection solution. Then, the beads were washed 10 times with Mtt deprotection solution and DMSO to obtain Ac-Lys beads and Ac-Cys beads.

\subsection{Procedure Using Ac-Lys Beads}

Ac-Lys beads ( $5 \mathrm{mg}$ in DMSO) were placed in two test tubes. One test tube was incubated with the test compound solution (in DMSO, $1 \% \mathrm{~N}$-methylmorpholine as a stabilizer of basic condition for the reaction) at $20-25^{\circ} \mathrm{C}$ for $24 \mathrm{~h}$ (test). The other test tube was incubated in DMSO containing $1 \%$ $\mathrm{N}$-methylmorphiline at $20-25^{\circ} \mathrm{C}$ for $24 \mathrm{~h}$ (control). After incubation, the beads were washed 5 times with DMSO and then with methanol $(\mathrm{MeOH})$. Following this, the two test tubes were incubated with $1 \mathrm{~mL}$ of $1 \mathrm{mM}$ bromophenol blue (BB) solution (in MeOH) for $30 \mathrm{~min}$ at $20-25^{\circ} \mathrm{C}$. Then, Ac-Lys beads were washed 10 times with $\mathrm{MeOH}$ to remove unreacted $\mathrm{BB}$. The beads were treated with $200 \mu \mathrm{L}$ saturated Lys solution for $5 \mathrm{~min}$ at $20-25^{\circ} \mathrm{C}$ to release the bound BB. After collecting the solutions from the two test tubes, absorbance $\left(\mathrm{OD}_{\text {test }}\right.$ and $\mathrm{OD}_{\text {control, }}$, respectively) of the solutions from test and control test tubes were measured at $591 \mathrm{~nm}$ using a UV spectrophotometer. 


\subsection{Calculation of Reactivity of Test Chemicals with Ac-Lys Beads}

Reactivity of Ac-Lys beads was expressed as percent depletion based on decrease in the amount of unreacted Lys after the reaction with the chemical, relative to the amount of Lys initially measured (Equation (1)).

$$
\text { Depletion ratio }(\%)=\left[1-\left(\mathrm{OD}_{\text {test }} / \mathrm{OD}_{\text {control }}\right)\right] \times 100
$$

where $\mathrm{OD}_{\text {control }}$ is the $\mathrm{OD}$ measured in the control solution in which the chemical was not added, and the OD test is the OD of the test solution in which the Ac-Lys beads were reacted with the chemical.

\section{Results and Discussion}

\subsection{Design and Synthesis of Lys-and Cys-Coupled Magnetic Beads}

We designed and synthesized Lys-and Cys-immobilized magnetic microbeads (Figure 1a). Micromer-M PEG- $\mathrm{NH}_{2}$ beads were selected because they are amphiphilic and swell in both hydrophilic and hydrophobic solvents. Fmoc-Lys(Mtt)-OH and Fmoc-Cys(Mtt)-OH, carrying acid-labile protecting group Mtt, were chosen as monomers for coupling because the magnetic beads are susceptible to strong acids. We used DMSO instead of N,N-dimethylformamide (DMF) and N-methylpyrrolidone (NMP), the commonly used solvents for Fmoc synthesis, because, unlike DMF and NMP, DMSO is a dispersion-stabilizing solvent for magnetic beads.

After optimizing the synthesis protocol [15], we assessed the purity of the beads loading the amino acids. We synthesized the dipeptide Fmoc-Lys(Mtt)-npp onto the magnetic microbeads (Figures S1 and S2, Supplementary Materials). The photo-labile npp linker [16,17] was used to release Fmoc-Lys(Mtt) moieties from the beads upon UV irradiation (Figure 2a). Following the coupling of Fmoc-npp-OH and Fmoc-Lys(Mtt)-OH and subsequent photo cleavage, the purity of the peptide was assessed using HPLC by monitoring the absorbance of the Fmoc moiety at $301 \mathrm{~nm}$. The HPLC chromatograph showed a peak at $15.5 \mathrm{~min}$, corresponding to the product Fmoc-Lys(Mtt)- $\mathrm{NH}_{2}$ (Figure 2b). Similarly, we also optimized and confirmed the Mtt deprotection protocol in DMSO. Following N-terminal deprotection and acetylation, the Mtt group on the sidechain was removed from the dipeptide and 5(6)-carboxyfluorescein was coupled to the free amine of the Lys side chain to obtain Ac-Lys(Flu)- $\mathrm{NH}_{2}$. After photo cleavage from the beads, we assessed the purity of the product by HPLC by monitoring the absorbance of the fluorescein moiety at $490 \mathrm{~nm}$. A clear peak at $14.5 \mathrm{~min}$ for the target product was obtained, indicating successful Mtt deprotection in DMSO (Figure 2c).

Using the above approach, we obtained Ac-Lys beads and Ac-Cys beads. However, Ac-Cys beads showed gradual decomposition of the magnetic beads due to $\mathrm{SH}$ oxidation (reduction of the magnetic beads). Therefore, only Ac-Lys beads were used in the following experiments. 
(a)

Fmoc-Lys(Mtt)-npp-beads

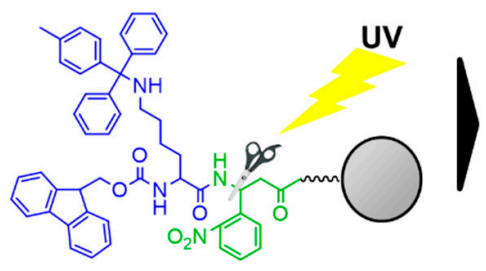

Ac-Lys(Flu)-npp-beads

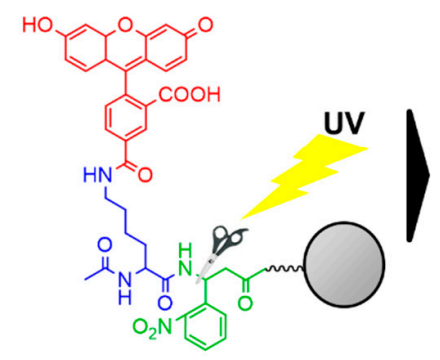

(b)

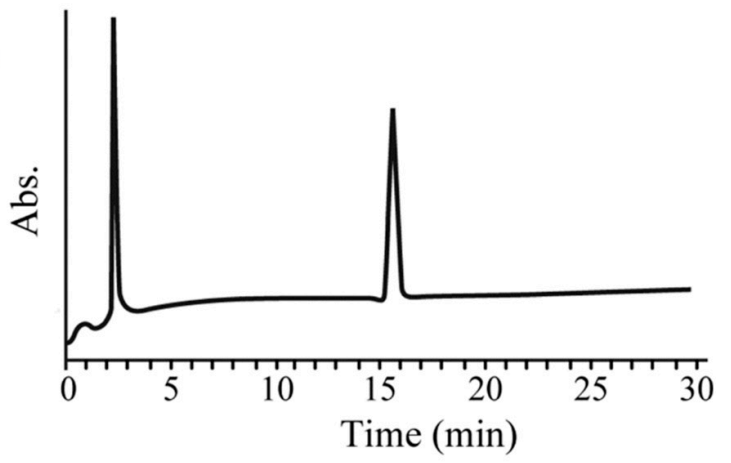

(c)

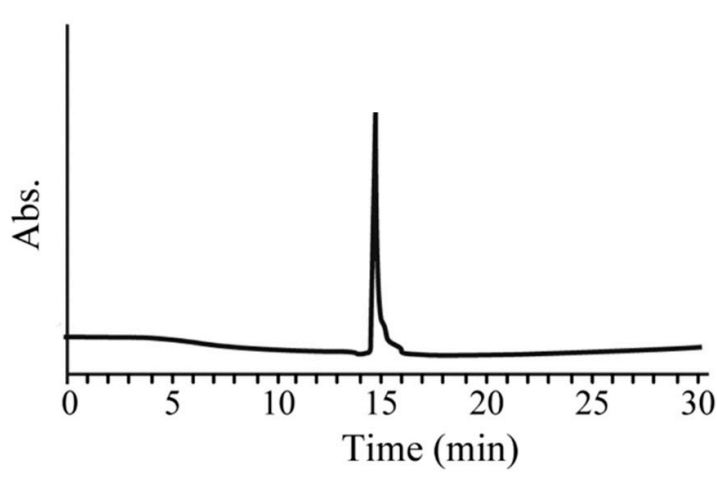

Figure 2. (a) Release of Fmoc-Lys(Mtt)- $\mathrm{NH}_{2}$ and $\mathrm{Ac}-\mathrm{Lys}(\mathrm{Flu})-\mathrm{NH}_{2}$ from the magnetic beads. HPLC chromatographs of Fmoc-Lys(Mtt)- $\mathrm{NH}_{2}$ (b) and Ac-Lys(Flu)- $\mathrm{NH}_{2}$ (c). HPLC was performed using an Inertsil ODS-3 column $(4.5 \times 150 \mathrm{~mm})$ for analysis with a linear gradient from $0 \%$ to $100 \%$ of solvent $\mathrm{B}$ (90\% acetonitrile, $10 \%$ Milli-Q water, and $0.08 \%$ trifluoroacetic acid (TFA)) over 30 min at a flow rate of $1.0 \mathrm{~mL} / \mathrm{min}$. The eluate was monitored at $301 \mathrm{~nm}$ for Fmoc moiety absorbance of Fmoc-Lys(Mtt)-NH or at $492 \mathrm{~nm}$ for fluorescein moiety absorbance of Ac-Lys(Flu)- $\mathrm{NH}_{2}$.

\subsection{C-SPRA-MB Using Ac-Lys Beads and FITC}

BB was used to detect and quantify the free amino groups on Ac-Lys beads, to evaluate both the reactivity of the Ac-Lys beads and the reactivity of the test chemicals (Figure 3a). BB forms a salt with the amine group on Ac-Lys beads and is released from the beads with dissociation of the ionic bond in an alkaline solution $[18,19]$. Additionally, the reactivity of BB was milder than that of picric acid used in C-SPRA [13], which generated side reaction with magnetic beads. Thus, measurement of the absorbance of $B B(591 \mathrm{~nm})$ offers quantification of the amount of amine on the beads spectrophotometrically. Unreacted BB was removed, and the reacted BB was released from the Ac-Lys beads using a saturated solution of lysine. The released BB was quantified. DMSO was suitable for the solvent in the reaction because DMSO can solve almost all the chemicals and was a dispersion-stabilizing solvent for magnetic beads. 
(a)

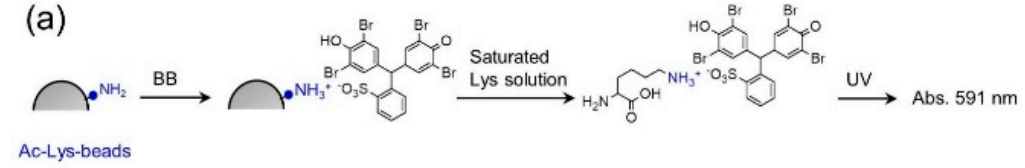

(b)
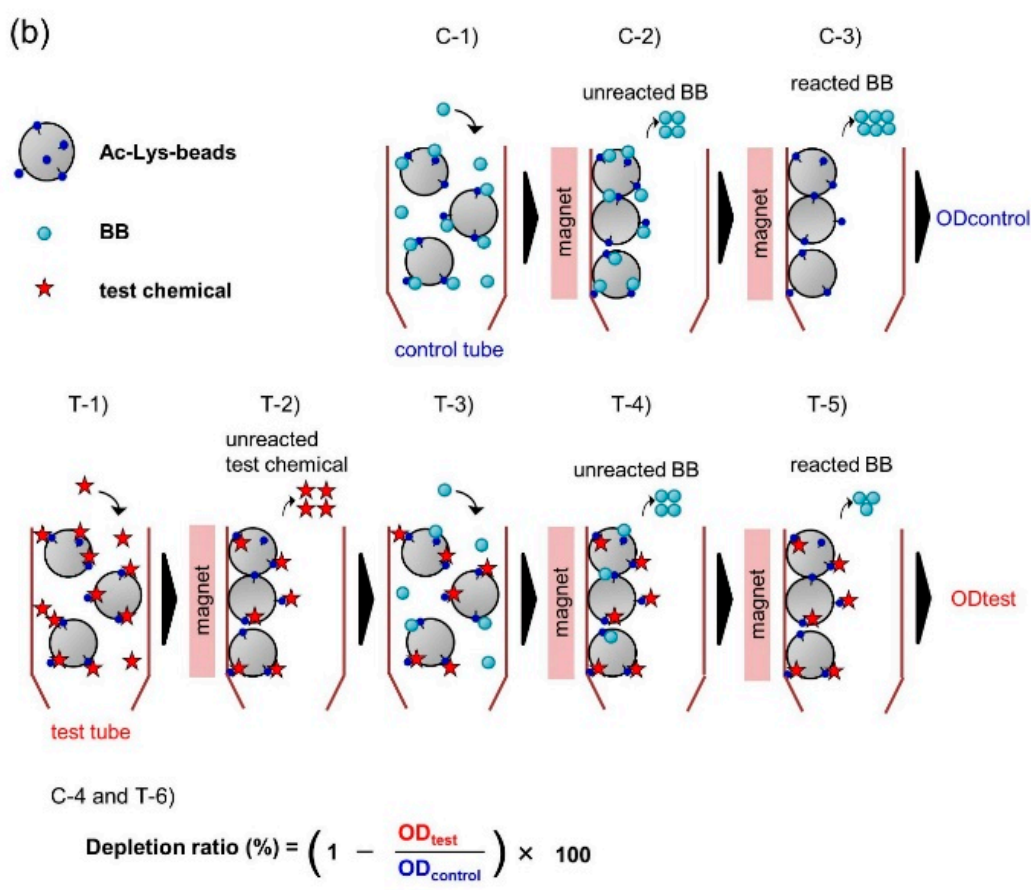

Figure 3. (a) C-SPRA-MB method with UV detection of bromophenol blue (BB). (b) Schematic representation of the procedures for using a magnet holder and separator in C-SPRA-MB and assessing skin sensitization. The procedure is as follows: (T-1) a test chemical was added; (T-2) after the reaction process $(24 \mathrm{~h})$, the Ac-Lys beads were washed; (C-1 and T-3) and the BB was added to Ac-Lys beads; (C-2 and T-4) the unreacted BB was removed out by washing with the solvent; (C-3 and T-5) the release solution (saturated Lys solution) was added for removal and collection of reacted chromophores, and $\mathrm{OD}_{\text {control }}$ and $\mathrm{OD}_{\text {test }}$ were obtained by UV measurement; (C-4 and T-6) the depletion ratio was calculated from $\mathrm{OD}_{\text {control }}$ and $\mathrm{OD}_{\text {test }}$.

We attempted to find suitable conditions of the measurement of Ac-Lys beads reactivity protocol as shown in Figure $3 b$ using FITC as a representative sensitizer. Percent depletion represented the reactivity of test chemicals to Ac-Lys beads. Conditions such as concentration of the test chemical and deration of reaction were evaluated to determine the suitable reaction protocols. Initially, we measured the dose-response curves of FITC to determine the suitable test chemical concentration. FITC depletion ratio increased in a dose-dependent manner, with a maximum depletion ratio at $100 \mathrm{mM}$, whereas at 1and 10-mM concentration of FITC low ratios were obtained (44\% and $46 \%$, respectively) (Figure $4 \mathrm{a}$ ). These results implied that C-SPRA-MB might assess potent sensitizers even in 1 and $10 \mathrm{mM}$ and that Ac-Lys beads can judge sufficiently to potent sensitizers at a concentration of $100 \mathrm{mM}$, which is the concentration of test chemical typically required for the conventional DPRA protocol. Therefore, we selected a test chemical concentration of $100 \mathrm{mM}$ in the following experiments. Further, we evaluated the reaction kinetics using $100 \mathrm{mM}$ FITC. As C-SPRA-MB using FITC showed time-dependent depletion ratios, C-SPRA-MB may be able to offer kinetic experiments with much shorter reactions than 1 or $3 \mathrm{~h}$. The maximum depletion ratio was observed at $24 \mathrm{~h}$ for FITC (Figure $4 \mathrm{~b}$ ), which is the same as the conventional DPRA and ADRA protocol. Thus, in the final protocol, Ac-Lys beads were incubated with $100 \mathrm{mM}$ test chemicals for $24 \mathrm{~h}$ at $20-25^{\circ} \mathrm{C}$. 
(a)

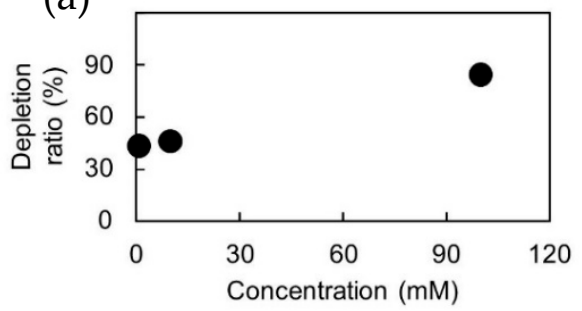

(b)

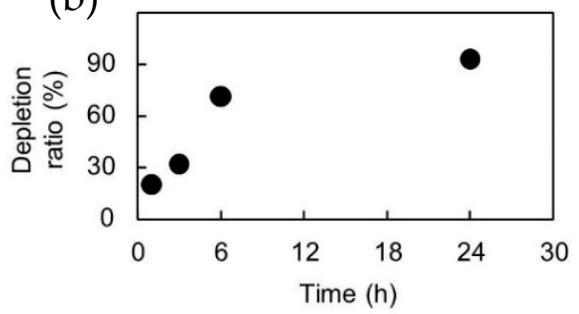

Figure 4. C-SPRA-MB using Ac-Lys beads and fluorescein-5-isothiocyanate (FITC). (a) Ac-Lys beads were tested with $1 \mathrm{mM}, 10 \mathrm{mM}$, and $100 \mathrm{mM}$ FITC for $24 \mathrm{~h}$. (b) Ac-Lys beads were examined at chemical concentrations of $100 \mathrm{mM}$ for $1,3,6$, and $24 \mathrm{~h}$.

\subsection{C-SPRA-MB Using Ac-Lys Beads}

For evaluation of the effectiveness and usefulness of C-SPRA-MB, we evaluated the reactivity of the Ac-Lys beads with eight representative test chemicals: an extreme sensitizer (BQ), a strong sensitizer (FITC), three moderate sensitizers (BA, UE, and MPH), a weak sensitizer (ACA), and a non-sensitizer (DP). Five of these test chemicals are poorly soluble in water, as their log Kow values are higher than 3.5 [20]. Among these, ACA is representative of chemicals detected as a false negative in conventional DPRA and ADRA (Table S1, Supplementary Materials). Table 1 shows the results of C-SPRA-MB, DPRA, and ADRA [6,21,22]. All chemicals were tested according to the scheme shown in Figure $3 \mathrm{~b}$. Depletion ratios over $30 \%$ were observed for all six sensitizers that were tested (BQ, FITC, BA, UE, MPH, and ACA) (Table 1), whereas conventional DPRA and ADRA could not assess hydrophobic chemicals such as ACA, which was identified as a false negative sensitizer. "P" denoted more than $20 \%$ mean depletion shown by test chemicals and " $\mathrm{N}$ " denoted less than $20 \%$ mean depletion shown by them (Table 1). The tested non-sensitizer (DP) showed a depletion ratio below $10 \%$, similar to the results of DPRA and ADRA. C-SPRA-MB could discriminate between all sensitizers and all non-harmful chemicals, using a threshold of about $20 \%$ for mean peptide depletion, which was ca. two times of negative depletion ratio (DP, $8.9 \%$ ) in this study. These results suggest that C-SPRA-MB is able to assess various sensitizers with poor water solubility, especially in the case of chemicals misclassified by DPRA and ADRA.

According to the in-silico analysis, UE and ACA were expected to react with proteins. Consequently, they should show positive results with our proposed test $[23,24]$. ACA has electron-deficient double bonds that are considered Michael acceptors due to susceptibility to nucleophilic attack [23,25]. UE is considered to react with the amine of Lys as an electrophile generating a Schiff base [25]. As expected, reactivity of these compounds was seen with Ac-Lys beads. Thus, C-SPRA-MB provided a higher mean percent depletion for sensitizers that were classified as false negatives by DPRA and ADRA. Usually, the test chemicals that DRPA returned as false negatives were found to be were sensitizers that were considered to be weakly potent, under in vivo assays including LLNA in the EURL ECVAM (European Union Reference Laboratory for alternatives to animal testing) study [12]. The false-negative chemicals such as ACA generally have poor water-solubility and they can exhibit moderate or weak sensitization potency in this study. Additionally, the moderate and weak sensitizers showed higher depletion ratios in this study than those in the previous study, C-SPRA [13]. It implied that these ratios resulted from easier accessibility of the chemicals to the C-SPRA-MB beads than that to C-SPRA resins. The possible reasons were as follows: (1) The peptide sequence was shorter than those in C-SPRA; (2) The peptides were immobilized on the surface of magnetic beads in C-SPRA-MB, whereas the peptides were immobilized to the resins from the surface to the inside in C-SPRA. Since the test chemicals can completely dissolve and react in the polar solvent in our method, C-SPRA-MB can predict various sensitizers even with poor water solubility. 
Table 1. Reactivity of 8 chemicals to Lys beads determined by percent depletion.

\begin{tabular}{|c|c|c|c|c|}
\hline \multirow[b]{2}{*}{ Test Chemicals } & \multirow{2}{*}{$\begin{array}{c}\text { Direct Peptide } \\
\text { Reactivity Assay } \\
\text { (DPRA) Results }{ }^{a, b}\end{array}$} & \multirow{2}{*}{$\begin{array}{l}\text { Amino Acid Derivative } \\
\text { Reactivity Assay } \\
\text { (ADRA) Results }{ }^{c}\end{array}$} & \multicolumn{2}{|c|}{ C-SPRA-MB } \\
\hline & & & $\begin{array}{l}\text { Depletion } \\
\text { Ratio (\%) }\end{array}$ & Results $^{d}$ \\
\hline$p$-Benzoquinone (BQ) & $\mathrm{P}^{\mathrm{a}}$ & $\mathrm{P}^{\mathrm{c}}$ & 92.5 & $\mathbf{P}$ \\
\hline Fluorescein-5-isothiocyanate (FITC) & $\mathrm{P}^{\mathrm{a}}$ & $\mathrm{P}^{\mathrm{c}}$ & 84.6 & $\mathbf{P}$ \\
\hline Benzylidene acetone (BA) & $\mathrm{P}^{\mathrm{a}}$ & $\mathrm{P}^{\mathrm{c}}$ & 75.0 & $\mathbf{P}$ \\
\hline 5-Methyl-2-phenyl-2-hexenal (MPH) & - & - & 46.2 & $\mathbf{P}$ \\
\hline Undec-10-enal (UE) & $\mathrm{P}^{\mathrm{b}}$ & - & 67.5 & $\mathbf{P}$ \\
\hline$\alpha$-Amyl cinnamic aldehyde (ACA) & $\mathrm{N}^{\mathrm{a}}$ & $\mathrm{N}^{\mathrm{c}}$ & 40.0 & $\mathbf{P}$ \\
\hline Dibutyl phthalate (DP) & $\mathrm{N}^{\mathrm{b}}$ & - & 9.7 & $\mathbf{N}$ \\
\hline
\end{tabular}

\section{Conclusions}

We achieved a synthetic method on magnetic microbeads and then a novel in vitro testing method, C-SPRA-MB, using Ac-Lys beads and chromophores to assess skin sensitizers. This approach demonstrated the utility of evaluation of chemicals with diverse sensitization potentials and poor water solubility. Additionally, we successfully optimized amino acid coupling to magnetic microbeads in DMSO. Our results indicate that C-SPRA-MB may be useful for effective prediction of their skin sensitization potential in the process of compound screening, especially in the case of misclassified by DPRA and ADRA. Furthermore, the reaction using immobilized peptides can be stopped at any point easily by washing, which allows for kinetics of the reaction to be followed easily. We will attempt to optimize the coupling of other amino acids to magnetic microbeads in DMSO in our future study and then this system, with substitution of the Ac-Lys bead sequence for some sequences-including the other amino acids, could further provide various information of sensitization. In addition, we should conduct validation experiments with a much larger number of compounds to assess their robustness and accuracy in our next study. C-SPRA-MB will hold promise as a representative next-generation sensitization test in vitro that will be able to address the disadvantages of conventional assessments. Although Ac-Cys beads could not assess skin sensitization potential due to self-reduction, we have already developed Cys-immobilized polystyrene beads [13]. Magnetic microbeads are easier to handle and are more versatile but have lower stability than polystyrene beads. Consequently, we can select magnetic microbeads or polystyrene beads in accordance to the intended use. Additionally, we showed an easy-cleavage process of amino acid from the beads and can combine this system with C-SPRA. This strategy may offer further detailed and important information including kinetics, reaction mechanisms, detailed reactivity, etc., by combining it with MS, HPLC, or NMR. This study expands C-SPRA applicability for assessing skin sensitizers without using animals for testing in food engineering, biochemical engineering, medicinal engineering, as well as cosmetic engineering. By assessing the sensitivities of various chemicals by C-SPRA-MB, we may better understand sensitization processes at the molecular level, which may also contribute to the developments in the field of biochemical engineering.

Supplementary Materials: The following are available online at http://www.mdpi.com/2227-9717/8/10/1257/s1, Figure S1: Structures of Fmoc-Lys(Mtt)-npp-beads and fluorescein tagged Ac-Lys(Flu)-npp-beads, Figure S2: Synthesis of Fmoc-Lys(Mtt)-npp-beads and Ac-Lys(Flu)-npp-beads, Table S1: Reactivity of test chemicals to Ac-Lys-beads determined by percent depletion, References.

Author Contributions: Conceptualization, H.M., H.A., K.Y. and K.U.; methodology, H.M., H.T., H.I. and Y.H.; writing-original draft preparation, H.M., K.Y. and K.U.; writing—review and editing, H.M., H.I., H.A., K.Y. and K.U. All authors have read and agreed to the published version of the manuscript. 
Funding: This research was funded by the Nipponham Foundation for the Future of Food (NFFF) and K.U. is grateful for Grants-in-Aid for Scientific Research (19K05741) from the Japan Society for the Promotion of Science (JSPS).

Acknowledgments: We would like to extend our thanks to Yasutaka Samejima (Konan University, Kobe, Japan) for technical assistance with the peptide synthesis and HPLC analysis.

Conflicts of Interest: The authors declare no conflict of interest.

\section{References}

1. Kimner, I.; Mitchell, J.A.; Griffin, A.C. Development of a murine local lymph node assay for the determination of sensitizing potential. Food Chem. Toxicol. 1986, 24, 585-586.

2. EU: Directive 2003/15/EC of the European Parliament and of the Council of 27 February 2003 amending Council Directive 76/768/EEC on the approximation of the laws of the Member States relating to cosmetic products (7th Amendment to the European Cosmetics Directive). Off. J. Eur. Communities Legis. 2003, 66, 26-35.

3. Kaplan, D.H.; Igyarto, B.Z.; Gaspari, A.A. Early immune events in the induction of allergic contact dermatitis. Nat. Rev. Immunol. 2012, 12, 114-124. [CrossRef] [PubMed]

4. Gerberick, G.F.; Vassallo, J.D.; Bailey, R.E.; Chaney, J.G.; Morrall, S.W.; Lepoittevin, J.P. Development of a peptide reactivity assay for screening contact allergens. Toxicol. Sci. 2004, 81, 332-343. [CrossRef]

5. Gerberick, G.F.; Vassallo, J.D.; Foertsch, L.M.; Price, B.B.; Chaney, J.G.; Lepoittevin, J.P. Quantification of chemical peptide reactivity for screening contact allergens: A classification tree model approach. Toxicol. Sci. 2007, 97, 417-427. [CrossRef]

6. Fujita, M.; Yamamoto, Y.; Tahara, H.; Kasahara, T.; Jimbo, Y.; Hioki, T. Development of a prediction method for skin sensitization using novel cysteine and lysine derivatives. J. Pharmacol. Toxicol. Methods 2014, 70, 94-105. [CrossRef]

7. Yamamoto, Y.; Tahara, H.; Usami, R.; Kasahara, T.; Jimbo, Y.; Hioki, T.; Fujita, M. A novel in chemico method to detect skin sensitizers in highly diluted reaction conditions. J. Appl. Toxicol. 2015, 35, 1348-1360. [CrossRef]

8. Fujita, M.; Yamamoto, Y.; Watanabe, S.; Sugawara, T.; Wakabayashi, K.; Tahara, K.; Horie, N.; Fujimoto, K.; Kusakari, K.; Kurokawa, Y.; et al. Cause of and countermeasures for oxidation of the cysteine-derived reagent used in the amino acid derivative reactivity assay. J. Appl. Toxicol. 2019, 39, 191-208. [CrossRef]

9. OECD. OECD Guidelines for testing of chemicals No. 442C. In Key-Event-Based Test Guideline for in Chemico Skin Sensitisation Assays Addressing the Adverse Outcome Pathway Key Event on Covalent Binding to Proteins; OECD Publishing: Paris, France, 2019.

10. Natsch, A.; Gfeller, H. LC-MS-based characterization of the peptide reactivity of chemicals to improve the in vitro prediction of the skin sensitization potential. Toxicol. Sci. 2008, 106, 464-478. [CrossRef]

11. Lalko, J.F.; Kimber, I.; Dearman, R.J.; Gerberick, G.F.; Sarlo, K.; Api, A.M. Chemical reactivity measurements: Potential for characterization of respiratory chemical allergens. Toxicol. In Vitro 2011, 25, 433-445. [CrossRef]

12. EURL-ECVAM. EURL ECVAM Recommendation on the Direct Peptide Reactivity Assay (dpra) for Skin Sensitisation Test; Publications Office of the European Union: Luxembourg, 2013.

13. Miyazaki, H.; Hamada, Y.; Takaishi, H.; Minamino, Y.; Ikeda, H.; Mekata, H.; Takaishi, M.; Yamashita, K.; Usui, K. Development of a chromophore-solid phase peptide reaction assay (C-SPRA) for assessing skin sensitization in vitro. Analyst 2020, 145, 3211-3216. [CrossRef] [PubMed]

14. Chan, W.C.; White, P.D. Fmoc Solid Phase Peptide Synthesis: A Practical Approach; Oxford University Press: Oxford, UK, 2000.

15. Usui, K.; Tomizaki, K.Y.; Mihara, H. A designed peptide chip: Protein fingerprinting technology with a dry peptide array and statistical data mining. Methods Mol. Biol. 2009, 570, 273-284. [PubMed]

16. Ariyasu, S.; Hanaya, K.; Watanabe, E.; Suzuki, T.; Horie, K.; Hayase, M.; Abe, R.; Aoki, S. Selective capture and collection of live target cells using a photoreactive silicon wafer device modified with antibodies via a photocleavable linker. Langmuir 2012, 28, 13118-13126. [CrossRef] [PubMed]

17. Usui, K.; Kikuchi, T.; Tomizaki, K.Y.; Kakiyama, T.; Mihara, H. A novel array format for monitoring cellular uptake using a photo-cleavable linker for peptide release. Chem. Commun. 2013, 49, 6394-6396. [CrossRef] [PubMed] 
18. Krchnak, V.; Vagner, J.; Lebl, M. Noninvasive continuous monitoring of solid-phase peptide synthesis by acid-base indicator. Int. J. Pept. Protein Res. 1988, 32, 415-416. [CrossRef] [PubMed]

19. Flegel, M.; Sheppard, R.C. A sensitive, general method for quantitative monitoring of continuous flow solid phase peptide synthesis. J. Chem. Soc. Chem. Commun. 1990, 7, 536-538. [CrossRef]

20. Takenouchi, O.; Miyazawa, M.; Saito, K.; Ashikaga, T.; Sakaguchi, H. Predictive performance of the human Cell Line Activation Test (h-CLAT) for lipophilic chemicals with high octanol-water partition coefficients. J. Toxicol. Sci. 2013, 38, 599-609. [CrossRef]

21. Natsch, A.; Ryan, C.A.; Foertsch, L.; Emter, R.; Jaworska, J.; Gerberick, F.; Kern, P. A dataset on 145 chemicals tested in alternative assays for skin sensitization undergoing prevalidation. J. Appl. Toxicol. 2013, 33, 1337-1352. [CrossRef]

22. Otsubo, Y.; Nishijo, T.; Miyazawa, M.; Saito, K.; Mizumachi, H.; Sakaguchi, H. Binary test battery with KeratinoSens ${ }^{\mathrm{TM}}$ and h-CLAT as part of a bottom-up approach for skin sensitization hazard prediction. Regul. Toxicol. Pharmacol. 2017, 88, 118-124. [CrossRef]

23. Roberts, D.W.; Patlewicz, G.; Kern, P.S.; Gerberick, F.; Kimber, I.; Dearman, R.J.; Ryan, C.A.; Basketter, D.A.; Aptula, A.O. Mechanistic applicability domain classification of a local lymph node assay dataset for skin sensitization. Chem. Res. Toxicol. 2007, 20, 1019-1030. [CrossRef]

24. Urbisch, D.; Mehling, A.; Guth, K.; Ramirez, T.; Honarvar, N.; Kolle, S.; Landsiedel, R.; Jaworska, J.; Kern, P.S.; Gerberick, F.; et al. Assessing skin sensitization hazard in mice and men using non-animal test methods. Regul. Toxicol. Pharm. 2015, 71, 337-351. [CrossRef] [PubMed]

25. Roberts, D.W.; Aptula, A.O.; Patlewicz, G. Electrophilic chemistry related to skin sensitization. Reaction mechanistic applicability domain classification for a published data set of 106 chemicals tested in the mouse local lymph node assay. Chem. Res. Toxicol. 2007, 20, 44-60. [CrossRef] [PubMed]

(C) 2020 by the authors. Licensee MDPI, Basel, Switzerland. This article is an open access article distributed under the terms and conditions of the Creative Commons Attribution (CC BY) license (http://creativecommons.org/licenses/by/4.0/). 